\title{
Wie über Hilfen zur Erziehung entschieden wird
}

\section{Die Haltungen einzelner Jugendämter sind so maßgebend wie änderbar}

Frank Eger

Die Zabl der Hilfen zur Erziehung ist zwischen einzelnen Jugendamtsbezirken sehr unterschiedlich. Neben Kriterien wie die Lebenslagen der Familien, politisch-fiskalische Einflussnahmen, bestehende Angebotsstrukturen der Jugendhilfe und demografische Aspekte spielt dabei auch der Wandel in der Organisation behördlicher Entscheidungsprozesse eine große Rolle.

Die öffentliche Jugendhilfe, auf kommunaler Ebene von Stadt- und Kreisjugendämtern repräsentiert, nimmt als Drehscheibe der Jugendhilfe nach wie vor eine zentrale Funktion in Fragen der Gewährung von Hilfen zur Erziehung ein. Äußeres Kennzeichen sind u. a. die dort zu treffenden Entscheidungen über die Hilfegewährung im Einzelfall. Das Endprodukt dieser Prozesse ist infolge der im Einzelfall gewährten Hilfeform zu erkennen, und die Gesamtheit der vonseiten eines Jugendamtes gewährten Hilfen spiegelt sich in den zu veröffentlichenden Fallzahlen wieder.

Die Gründe für die je nach Jugendamtsbezirk unterschiedliche Inanspruchnahme einzelner Hilfeformen sind jedoch seit jeher Anlass für Kontroversen (vgl. LWV 1998: 3 ff). Der Fokus bisheriger Untersuchungen zu dem Thema war bisher vornehmlich auf sozialstrukturelle Verhältnisse, Angebotsstrukturen und demografische Merkmale gerichtet. Dem Wandel und der Durchsetzung organisationaler Merkmale im Hinblick auf jugendamtliche Entscheidungsprozesse wurde demgegenüber bisher nur in geringem Maße Aufmerksamkeit zuteil.

Prof. Dr. Frank Eger lehrt an der Fachhochschule Braunschweig/ Wolfenbüttel in der Fakultät Soziale Arbeit.

E-Mailf.eger@ostfalia.de
Eger (2008) hat zu diesem Thema mit einer Organisationsuntersuchung in mehreren Beispielen die Lernprozesse eines kommunalen Jugendamtes aus dem nördlichen Rheinland-Pfalz nachgewiesen. Die bisherigen Untersuchungen verweisen übereinstimmend auf eine Multikausalität, mithin eine Vielzahl der Ursachen für die je unterschiedliche Inanspruchnahme von Hilfen zur Erziehung (vgl. LWV 1998: 23). Als maßgeblich werden insbesondere die sozialen Verhältnisse, also Lebenslagen der Familien, politisch-fiskalische Einflussnahmen, bestehende Angebotsstrukturen der Jugendhilfe und demografische Aspekte bewertet.

\section{Sozialstrukturelle Bedingungen}

Auf die Bedeutung sozialstruktureller Bedingungen für die Inanspruchnahme von Hilfen zur Erziehung wurde in einer Vielzahl von Studien hingewiesen. Mit den Hilfen zur Erziehung werden in der Hauptsache Kinder, Jugendliche und Familien betreut, die aus belasteten Familiensituationen kommen. Auch die Verfasser des »1. Landesberichts Hilfen zur Erziehung in Rheinland-Pfalz (MASFG 2004: 198) kommen zu ähnlichen Ergebnissen hinsichtlich der Inanspruchnahme von Hilfen zur Erziehung in ihrer Gesamtheit in dem Bundesland: "Der Tendenz nach steigt die Inanspruchnahme von Hilfen zur Erziehung, wenn sich die soziostrukturellen Rahmenbedingungen einer Kommune verschlechtern.«

\section{Politisch-fiskalische Einflussnahmen}

Diese Kategorie verweist auf Vorgaben, die aus kommunalpolitischen Gremien oder seitens der Verwaltungsspitze steuernden Einfluss darauf nehmen, in welchem Umfang Hilfen gewährt werden können. Die viel diskutierten (u. a. Herwig-Lempp 2008) Vorgänge in der Stadtverwaltung Halle stehen dafür als ein Beispiel unter vielen. Das Feld der Hilfen zur Erziehung ist in besonderem Maße anfällig für politische Steuerung, da entsprechende Vorgaben oftmals keinen Aufschrei der Adressaten auslösen.

\section{Wechselwirkung zwischen stationären und nicht- stationären Erziehungshilfen}

Das Institut für Sozialpädagogische Forschung Mainz e. V. (ism Mainz) hat in einer interkommunalen Vergleichsstudie festgestellt: "Ambulante und teilstationäre Hilfen wirken bedarfsmindernd auf Fremdunterbringungen. " (MASFG 2004) Ob dieser Effekt eintritt, hängt insbesondere mit konzeptionellen Merkmalen ambulanter und teilstationärer Hilfen zusammen (Eger 2008). Insbesondere die Zugangsschwelle zu ambulanten und teilstationären Hilfen ist ein für die Bedarfsminderung bei den stationären Hilfen maßgeblicher Faktor.

\section{Demografische Entwicklung}

Die demografische Entwicklung ist auch ein für die Bedarfsentwicklung bei den Hilfen zur Erziehung relevanter Faktor. So werden ab dem Jahr 2010 aufgrund des demografischen Wandels diejenigen Altersgruppen deutlich abnehmen, die am stärksten die Nachfrage nach Hilfen zur Erziehung prägen (MASFG 2004: 198).

\section{Haltungen der Jugendämter}

Die bisherigen Ausführungen geben bereits zu erkennen, dass die Gewährleistung der Hilfen zur Erziehung äußerst komplexen Faktoren unterliegt. Frühe 
Untersuchungen der Autoren des LWV Württemberg-Hohenzollern (1998: 17) verweisen darüber hinaus auf die mögliche Relevanz der "Arbeitsweisen und Arbeitsbedingungen « in Jugendämtern und subsumieren darunter die Bedeutung unterschiedlicher Perspektiven (Jugendamtsperspektive, Sichtweise der Eltern, Teamstrukturen); gleichzeitig weisen die Autoren (LWV 1998: 13) jugendamtlichen Definitionsprozessen eher eine sekundäre Bedeutung bei:

»Die herausragende Bedeutung der sozialstrukturellen Bedingungen darf nun natürlich nicht so verstanden werden, dass andere Einflussfelder - etwa Definitions- und Entscheidungsprozesse in einem Jugendamt - deshalb bedeutungslos wären. Bemerkenswert ist aber dies: Offensichtlich liegt hinter solchen Prozessen eine Folie harter sozialer Wirklichkeit, die den Bedarf an Fremdunterbringungen in erster Linie beeinflusst. Diese Erkenntnis verweist auch darauf, dass der gelegentlich anzutreffende Verdacht, die Jugendhilfe definiere eher leichtfertig und nach eigenem Gusto ihren Handlungsbedarf, kaum der Realität entspricht. «

Die Autoren (LWV 1998: 17) konnten dann auch infolge dieser als "schillernd « bezeichneten Versuche einer stringenten Durchsetzung jugendamtlicher Perspektiven bezogen auf die Gesamtheit der untersuchten 22 Jugendämter keinen Hinweis auf einen Zusammenhang zwischen Aushandlungsstrategien und Fremdunterbringungseckwerten der Kreise feststellen.

Seit einigen Jahren hat sich die Aufmerksamkeit in der Fachdiskussion jedoch hin zu Fragen der Konstruktion von Bedarfslagen verschoben. Merchel (2003: 4) stellt dazu fest: »Die Art und Weise jedoch, wie Akteure mit diesen sozialstrukturellen Bedingungen umgehen, in welcher Weise sie diese wahrnehmen und welche Schlussfolgerungen für Handlungen sie aus diesen Wahrnehmungen ableiten, kann, wie wir aus einer einfachen Betrachtung des Handlungsalltags in unterschiedlichen Regionen wissen, sehr verschiedenartig sein. «

Eger (2008) hat Lernprozesse eines kommunalen Jugendamtes in den Jahren 1992-2002 nachgewiesen, die auf eine Änderung unterschiedlicher Haltungen sowie Arbeitsweisen und Arbeitsbedingungen schließen lassen, die nicht zuletzt für Entscheidungen um Hilfen zur Erzie- hung ihre Relevanz erweisen. Mit dem jugendamtlichen Lernen ging eine deutliche Veränderung der Inanspruchnahme von Hilfen zur Erziehung einher: von einer anfänglich überwiegenden Gewährung von stationären zu ambulanten und teilstationären Hilfen.

Die veränderten Wissensbestandteile äußern sich in einem Bewusstsein um eigene Steuerungspotentiale, der Lokalisierung von Entscheidungskompetenz, der Regionalisierung sozialer Dienste, der Zugangsschwelle ambulanter Hilfen zur Erziehung sowie in Konflikten um die Deutungshoheit bei den Hilfen zur Erziehung.

\section{Entdeckung eigener Steuerungspotentiale}

Die Frage um die Durchsetzung der Jugendamtsperspektive ist unmittelbar mit dem Aspekt der Steuerung zu verbinden. Merchel (2003: 4) betont zwar, dass die zu beeinflussenden Faktoren viel zu komplex und beweglich seien, als dass eine "sichere "Vorhersage von Ursache und Wirkung erfolgen könne. Sobald die Steuerung der Hilfen zur Erziehung thematisiert werde, gehe es immer um strategische Entscheidungen, deren Auswirkungen nicht sicher vorhersagbar seien. Der Autor (2003: 5) weist darauf hin, dass die handelnden Akteure in der Jugendhilfe auf diesen Aspekt des Glaubens an die eigenen Steuerungsmöglichkeiten trotzdem nicht verzichten würden. Ganz im Gegenteil sei »die Versuchung groß, Zweifel um die Technisierbarkeit der Steuerung in personverändernden Handlungsfeldern zu umgehen und stattdessen die vermeintliche Wirkung des eigenen Handelns in den Vordergrund der Betrachtung zu rücken «.

Die Untersuchung von Eger (2008) über den Wandel der Inanspruchnahme von Hilfen zur Erziehung eines kommunalen Jugendamtes unterstützt Merchels These. Die Ergebnisse zeigen beispielsweise, dass in dem untersuchten Jugendamt zunächst die Position dominierte, dass die Anzahl der Kinder und Jugendlichen in Heimerziehung Resultat schicksalhafter, personenbezogener Entwicklungen ist. Nachdem jedoch eine vermeintlich hohe Anzahl Minderjähriger aus fachlichen und finanziellen Gründen aus dem politischen Raum kritisiert wur- de, änderte die Verwaltung die Ursachenzuschreibung. Die darauf folgende Senkung der Fallzahlen Heimerziehung wurde auf den Ausbau ambulanter Hilfen zur Erziehung zurückgeführt.Das Jugendamt lernte also, die Höhe der Fallzahlen nicht als schicksalhaft bedingt, sondern als Folge der Steuerungsbemühungen der Organisation zu bewerten.

\section{Wer über Hilfen zur Erziehung entscheidet}

Die Verwaltungen der Jugendämter nehmen im Rahmen des auf den Einzelfall bezogenen Hilfeplanverfahrens zentrale Verantwortung wahr. In den unterschiedlichen Phasen des Verfahrens sind formelle Voraussetzungen $\mathrm{zu}$ beachten. So ist beispielsweise gemäß $\mathbb{3} 3$ Abs. 2 SGB VIII die Entscheidung über die im Einzelfall angezeigte Hilfeart im $\mathrm{Zu}$ sammenwirken mehrerer Fachkräfte zu treffen.

Die Untersuchung offenbarte Widerspruch und Lösungsversuche zwischen den Ansprüchen der Linienorganisation und fachlich-pädagogischen Standards. Das untersuchte Jugendamt stellte mit der anfänglichen Bestimmung, dass die Entscheidung über die Gewährung von Hilfen zur Erziehung von einer Person gefällt wird, Verbindlichkeit her. Diese Entscheidung bedeutete gleichzeitig eine örtliche Asymmetrisierung, d. h. die Lokalisierung der Entscheidungskompetenz, in diesem Fall bei einer Person. Darüber hinaus verzichtete die Organisation in diesem anfänglichen Stadium auf eine inhaltliche Asymmetrisierung, beispielsweise im Hinblick auf die Fachlichkeit von Entscheidungsverfahren.

Im Weiteren verlagerte das Jugendamt jedoch die Entscheidungszuständigkeit von einer Person auf ein Team. Die somit neu lokalisierte Zuständigkeit wurde diesmal inhaltlich mittels Zielen begründet. Ziele als mögliches Element inhaltlicher Asymmetrisierung stellen den Versuch dar, die Selektivität des Systems zu konkretisieren und gleichzeitig weiter zu stabilisieren. Im vorliegenden Beispiel wurde also die Erwartung konkretisiert, unter Maßgabe bestehender Rahmenbedingungen Fachlichkeit zu gewährleisten. Ziele (hier: Fachlichkeit) legen damit nicht nur die Bedingungen für die Richtigkeit einer einzelnen Entscheidung fest; 
durch sie entstehen Pfade, nach denen ganze Entscheidungsketten ablaufen können.

Die Organisation lernte somit, dass infolge der inhaltlichen und örtlichen (Neu-) Bestimmung der Entscheidungsverantwortung Ziele langfristig realisiert werden können. Entscheidungskompetenz blieb nicht an eine Person singulär gebunden, sondern wurde mit dem Führungsteam auf eine breitere Basis gestellt. sation Vorstellungen einer zentralistischen Organisation auch des Sozialen Dienstes entsprechend dem Bedürfnis strikter Kopplung der Interaktionsebene an die Administrative. In der Fachdiskussion erfolgte zu der damaligen Zeit deutliche Kritik an bürokratisch organisierter Sozialer Arbeit (Ämterhierarchie, Arbeitsteilung u. a.). Stattdessen erarbeiteten Kommunale Gemeinschaftsstelle für Verwaltungsmanagement (KGSt) und Deut-

\section{»Hilfeformen lassen sich nach ihrer Schwellenhöhe für die Klienten unterscheiden "}

\section{Aufbauorganisation in Bewegung}

Organisationen in der Jugendhilfe müssen auf den Umstand reagieren, dass ihnen die Bedingungen, unter denen sie ihre Autonomie auszuüben vermögen, von der Gesellschaft entzogen werden können. Vor diesem Hintergrund wird die These nachvollziehbar, dass der Bestand einer Organisation von einer pauschalen gesellschaftlichen Bestätigung der Notwendigkeit ihrer Produkte abhängig ist (vgl. Kuper 2004: 145). Dahinter verbergen sich komplexe Aushandlungsprozesse, die Organisationen in der Jugendhilfe mit ihrer Umwelt um Ressourcensicherung und Leistungserstellung führen.

Organisationsberatung ist ein möglicher Beobachtungs- und Irritationsmodus, mit dem beispielsweise das Verhältnis von Ressourcen auf der einen Seite und Leistungserbringung auf der anderen Seite einer Prüfung unterzogen werden kann. Eben dieses Modus bediente sich das zur Untersuchung stehende kommunale Jugendamt. Externe Organisationsberater erhielten den Auftrag, die untersuchten Dienststellen der inneren Struktur und technischen Ausstattung neuesten Organisationskenntnissen anzupassen, Aussagen zum Personalbedarf zu treffen sowie den Bürgerservice weiter zu optimieren.

Die Organisationsberater nahmen u. a. auf die sozialräumliche Zuordnung der Aufgaben des Allgemeinen Sozialen Dienstes Bezug. Zu Beginn vertrat die Organi- scher Verein Vorschläge, die u. a. darauf zielten, soziale Dienste wohngebietsbezogen, umfeld- und teamorientiert zu organisieren. Das Prinzip der Teamarbeit zielte anstelle der Hierarchie auf kollegiale Arbeitsbeziehungen, um die Verantwortung für schwierige und weitreichende Entscheidungen der Gruppe zu übertragen.

Nach und nach war das untersuchte Jugendamt bereit, dezentrale Formen der Arbeitsorganisation für die sozialen Dienste zu entwickeln und damit tradierte Organisationsgrundsätze aufzugeben, um primär sozialpädagogisch-fachliche und betriebswirtschaftliche Ziele zu erreichen. In dem Beispiel lernte die Organisation, dass Elemente einer dezentralen Aufbauorganisation aus fachlichen und monetären Gründen angemessen sein können.

\section{Veränderungen von Zugangsschwellen}

Die Operative ist der Bereich innerhalb von Organisationen, in dem deren Prämissen praktisch umgesetzt werden. Deren Umsetzung erfordert von den Akteuren die situative Beurteilung bestimmter Ereignisse im Lichte einer vorgängig aufgestellten Entscheidung. Wenn von dem Mitarbeiter einer Verwaltung also erwartet werden kann, dass seine Rolle in der Operative durch die Mitgliedschaft in der Organisation bestimmt ist, so gehört zu dieser Rolle gleichzeitig die Entwicklung von Zufallssensibilitäten für unvorherge- sehene Ereignisse, die Auswirkungen auf die Anwendbarkeit der Deutungsmuster haben.

Der Mitarbeiter im Allgemeinen Sozialdienst muss also im Anschluss an die Feststellung eines möglichen Bedarfs klären, ob erstens für eine Hilfe zur Erziehung Kapazitäten zur Verfügung stehen und zweitens, ob die Organisation dem Klienten für diese Hilfeform einen Zugang ermöglicht.

In diesem Zusammenhang wird oftmals der Begriff der Niederschwelligkeit bemüht. Denn ebenso wie in anderen Bereichen der Sozialarbeit lassen sich auch in der Jugendhilfe die Hilfeformen nach der "Schwellenhöhe « differenzieren. Die Schwelle markiert dabei die Voraussetzungen, die Klienten erfüllen müssen, um ein Angebot in Anspruch nehmen zu können. Im Hinblick auf die Sozialpädagogische Familienhilfe wurde für den Jugendamtsbezirk des zur Untersuchung stehenden Jugendamtes der Zugang an die Voraussetzung gebunden, dass diese ambulante Hilfeform nicht als letzte Möglichkeit vor einer Fremdunterbringung oder einem Sorgerechtsentzug zur Anwendung kommt. Genau diese Schwellenhöhe verhinderte jedoch zunächst eine umfassende Bedarfsdeckung innerhalb des Jugendamtsbezirks. Die Zielsetzung eines Vorrangs familienergänzender Maßnahmen vor Herausnahme und Fremdunterbringung war nur infolge einer Senkung der Schwellenhöhe für den Zugang zur Sozialpädagogischen Familienhilfe möglich. Eine entsprechende Veränderung im Sinne einer Niederschwelligkeit wurde dann umgesetzt.

Die Organisation lernte also vorliegend, dass der Vorrang ambulanter Hilfen gegenüber Fremdunterbringungen eine Senkung des Zugangsniveaus aufseiten der erstgenannten Hilfeform erfordert.

\section{Wer die Deutungshoheit erhält}

Für die Ausprägung des organisationsspezifischen Kommunikationsbereichs ist in hohem Maße die Zugehörigkeit der Organisation zu einem gesellschaftlichen Teilsystem von Relevanz. Mit der Eingliederung einer Organisation in ein Teilsystem erfolgt die Ausrichtung nach ihrer Funktion sowie die Zuordnung zu dessen Kommunikationsbereich. 
Das untersuchte Jugendamt folgte im Hinblick auf die Deutung geeigneter und notwendiger Hilfen zur Erziehung zu Beginn des Lernprozesses zunächst noch weitgehend der argumentativen Begründung aus dem medizinischen Kommunikationsbereich. Die Ausgangslage für ein organisationales Gesichtswahren (»Facette «) gegenüber der gesellschaftlichen Umwelt sowie die Steuerung des operationalen Bereichs verweisen im vorliegenden Beispiel zunächst auf eine Indifferenz sozialpädagogischer Positionen gegenüber Vorgaben aus dem medizinischen Teilsystem.

In der Folge war das untersuchte Jugendamt jedoch in der Lage, die geeignete und notwendige Hilfe nicht mehr an die Empfehlung der Mediziner zu koppeln, sondern erhielt mit der Entwicklung von Programmen (beispielsweise mit einer Konzeptionen über sozialpädagogische Diagnostik) eigene Bezugsgrößen. Mit der Entscheidung für ein Programm wird eine Abgrenzung zu anderen Kommunikationsbereichen ermöglicht und gleichzeitig eine organisationsintern verfügbare Orientierungsgrundlage erstellt, die in den Entscheidungen fortlaufend mit den Absichten der Jugendhilfe und den Bestandsvoraussetzungen der Organisation in Abstimmung gebracht wird.
Die Organisation lernte in der Auseinandersetzung mit dem Einzelthema, sich in Fragen der Deutung geeigneter und notwendiger Hilfen gegenüber den organisierten Systemen eines anderen gesellschaftlichen Teilbereichs, hier den Empfehlungen der Ärzte der Kinder- und Jugendpsychiatrie, mithin des medizinischen Systems, abzugrenzen.

\section{Schluss}

Die Beispiele weisen Lernprozesse eines Jugendamtes nach. Gleichzeitig offenbaren diese die Dominanz von Organisationsinteressen im Hinblick auf Entscheidungsprozesse bei den Hilfen zur Erziehung. Das administrativ bei den Hilfen zur Erziehung orientierte Jugendamt zeigt nicht zuletzt, dass es die eigenen Steuerungsmöglichkeiten internalisiert hat.

Demgegenüber geraten Verfahren, die die Stimme der Adressaten zu Gehör bringen, in den Hintergrund. Dazu bedarf es einer Haltung, die den dialogischen Prozess zentral stellt und gemeinsam mit Kindern, Jugendlichen und Familien Anschlüsse für einen Bildungsprozess in den Hilfen zur Erziehung erarbeitet. Genau diese Themen bieten Anlass für weitere Lernprozesse des betreffenden Jugendamtes.

\section{Literatur}

Eger, Frank (2008): wie Jugendämter entscheiden. Ursachen einer veränderten Inanspruchnahme von Hilfen zur Erziehung. Wiesbaden: VS Verlag für Sozialwissenschaften.

Hansbauer, Peter (1999): Traditionsbrüche in der Heimerziehung: Analysen zur Durchsetzung der ambulanten Einzelbetreuung. Münster: Votum.

Herwig-Lempp, J. (2008): Schlecht beraten: In Halle regiert der »Roth-Stift «, in: Forum Sozial, $1 / 2008$.

LWV (Hg.) (1998): Ursachen der unterschiedlichen Inanspruchnahme von Heimerziehung. Zusammenfassung zentraler Ergebnisse der »Eckwertuntersuchung «. Stuttgart: LWV.

Luhmann, Niklas (2000): Organisation und Entscheidung, Opladen und Wiesbaden: Westdeutscher Verlag.

MASFG (Hg.) (2004): Hilfen zur Erziehung in Rheinland-Pfalz. Die Inanspruchnahme von Hilfen zur Erziehung im Kontext sozio- und infrastruktureller Einflussfaktoren. 1. Landesbericht. Mainz: MASFG.

Merchel, Joachim (2005): Organisationsgestaltung in der Sozialen Arbeit. Grundlagen und Konzepte zur Reflexion, Gestaltung und Veränderung von Organisationen. Weinheim und München: Juventa.

Willke, Helmut (1995): Systemtheorie III: Steuerungstheorie. Stuttgart: UTB.

\section{Aktuelle Neuerscheinung}

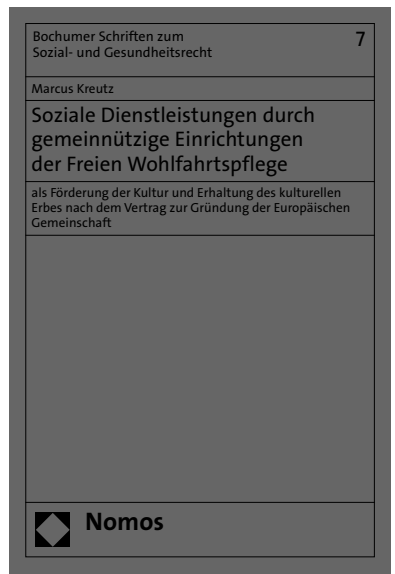

\section{Soziale Dienstleistungen durch gemeinnützige Einrichtungen der Freien Wohlfahrtspflege} als Förderung der Kultur und Erhaltung des kulturellen Erbes nach dem Vertrag zur Gründung der Europäischen Gemeinschaft Von RA Dr. Marcus Kreutz, LL.M.

2010, 362 S., brosch., 85,-€, ISBN 978-3-8329-5418-5

(Bochumer Schriften zum Sozialund Gesundheitsrecht, Bd. 7)

Nationale Privilegien, die der Gesetzgeber gemeinnützigen Körperschaften gewährt, sind nicht zwingend als verbotene Beihilfe im Sinne von Art. 87 Abs. 1 EGV zu qualifizieren. Vielmehr ergibt sich, dass diese Privilegien als Kultur und Erhaltung des kulturellen Erbes über Art. 87 Abs. 3 lit. d) EGV rechtfertigungsfähig sind.

\section{Nomos}

